\title{
Masked Tumor: A Rare Presentation of Struma Ovarii Disguised under Features Imitating Malignancy
}

\author{
Suhani Chandra ${ }^{1}$, Shraddha Shetty ${ }^{2}$, Vatsala Mallya ${ }^{3}$, Rashmi Polnaya ${ }^{4}$, Suprajna Shetty ${ }^{5}$, Cheryl Philipose ${ }^{6}$
}

\begin{abstract}
Aim: To present a rare case of struma ovarii in a 46-year-old lady presenting with features pointing toward malignancy.

Background: Struma ovarii is a specialized or monodermal teratoma, which is predominantly composed of mature thyroid tissue. It accounts for less than $5 \%$ of all ovarian teratomas. It is mostly benign, and malignant changes are extremely rare. Most patients are asymptomatic or may present with mild signs and symptoms. Although the vast majority are benign, they may rarely present mimicking malignancy.

Case description: A 46-year-old lady presented with a large complex abdominopelvic mass with ascites and elevated CA-125 levels, thus arising suspicion of malignancy. Postoperatively, histopathology revealed a benign struma ovarii, which was further confirmed by immunohistochemistry. Conclusion: Struma ovarii can imitate ovarian malignancy clinically, especially if it is complex and found in combination with ascites and an elevated CA-125 level. There is paucity of literature about such cases, and hence, it makes an accurate and timely diagnosis difficult.

Clinical significance: This case is one of the very few that provide a description of an atypical presentation of a rare tumor, struma ovarii. A high level of clinical suspicion may help with better preparedness before surgery and prevent extensive laparotomies in such patients.

Keywords: Germ cell tumors: case report, Hyperthyroidism, Mature teratoma, Monodermal teratoma, Ovarian tumor, Struma ovarii. Journal of South Asian Federation of Obstetrics and Gynaecology (2020): 10.5005/.jp-journals-10006-1831
\end{abstract}

\section{BACKGROUND}

Struma ovarii is a specialized mature teratoma mostly comprising of mature thyroid tissue. The tissue of thyroid origin must make up at least $50 \%$ of the entirety to be designated as a struma ovarii. It is an exceedingly rare histopathological diagnosis and is found in just $3 \%$ of ovarian teratomas, $2 \%$ of all germ cell tumors, and less than $1 \%$ of all ovarian tumors. It is most often found associated with a mature cystic teratoma, rarely with a cystadenoma. Most cases are benign and can be managed by surgical excision. Malignant change is extremely rare. ${ }^{1}$ Ascites may be found in approximately $17 \%$ of cases, and association with elevated CA-125 is rare. ${ }^{2}$ We hereby present a case of struma ovarii that presented with misleading findings pointing toward malignancy.

\section{Case Description}

A 46-year-old lady reported to our hospital with a history of abdominal distension for the last 1 month. On per abdomen examination, a mass of approximately $10 \times 8 \mathrm{~cm}$ was noted arising from the pelvis. It was nontender, had solid to cystic inconsistency, and was transversely mobile. On bimanual examination, a large cystic mass was felt posterior to and separate from the uterus. The ultrasonographic evaluation revealed moderate ascites and a large cystic mass of $12 \times 6 \mathrm{~cm}$ in the pelvis with septations within. CA-125 was found to be 270 . All other blood investigations were within normal limits. Total abdominal hysterectomy with $\mathrm{B} / \mathrm{L}$ salpingoophorectomy with infracolic omentectomy was performed with a high degree of suspicion for malignancy. Intraoperative findings revealed gross serous ascites. A mass of $11 \times 8 \times 4 \mathrm{~cm}$ with solid and cystic components arising from the left ovary was noted and removed (Fig. 1). Histopathology report revealed a monodermal teratoma-struma ovarii, with negative fluid cytology (Fig. 2A), Thyroid transcription factor 1 (TTF-1) immunohistochemistry marker was detected to be positive, further confirming the diagnosis (Fig. 2B).

\footnotetext{
${ }^{1-5}$ Department of Obstetrics and Gynecology, Kasturba Medical College, Mangaluru, Manipal Academy of Higher Education, Karnataka, India

${ }^{6}$ Department of Pathology, Kasturba Medical College, Mangaluru, Manipal Academy of Higher Education, Karnataka, India
}

Corresponding Author: Shraddha Shetty, Department of Obstetrics and Gynecology, Kasturba Medical College, Mangaluru, Karnataka, India, Phone: +919886792043, e-mail: drshraddhashetty@gmail.com

How to cite this article: Chandra S, Shetty S, Mallya V, et al. Masked Tumor: A Rare Presentation of Struma Ovarii Disguised under Features Imitating Malignancy. J South Asian Feder Obst Gynae 2020;12(6):436-437.

Source of support: Nil

Conflict of interest: None

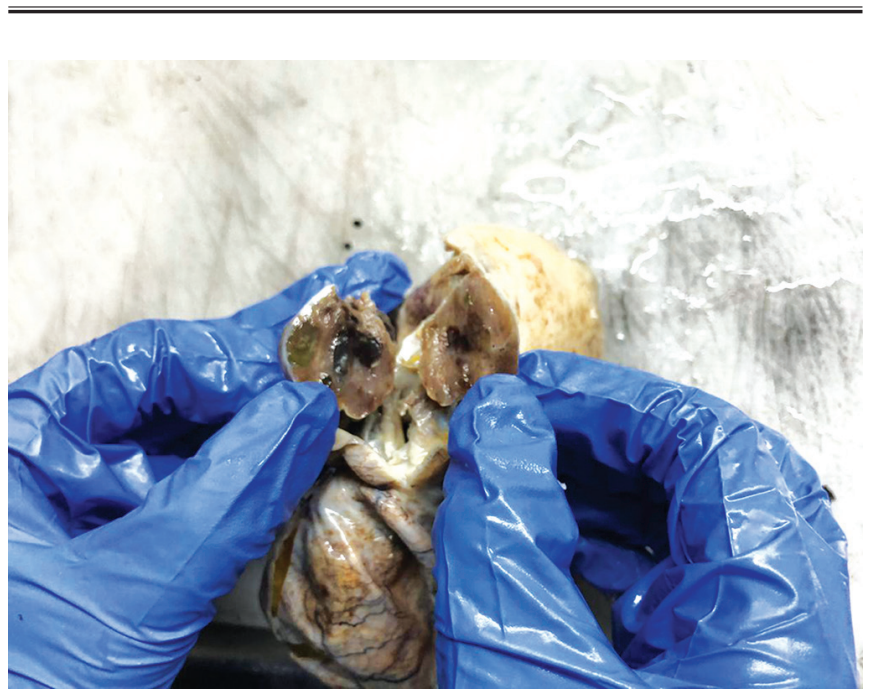

Fig. 1: Cut section of struma ovarii nodule showing pale brown, gelatinous material with areas of hemorrhage

(0) Jaypee Brothers Medical Publishers. 2020 Open Access This article is distributed under the terms of the Creative Commons Attribution 4.0 International License (https://creativecommons.org/licenses/by-nc/4.0/), which permits unrestricted use, distribution, and non-commercial reproduction in any medium, provided you give appropriate credit to the original author(s) and the source, provide a link to the Creative Commons license, and indicate if changes were made. The Creative Commons Public Domain Dedication waiver (http://creativecommons.org/publicdomain/zero/1.0/) applies to the data made available in this article, unless otherwise stated. 

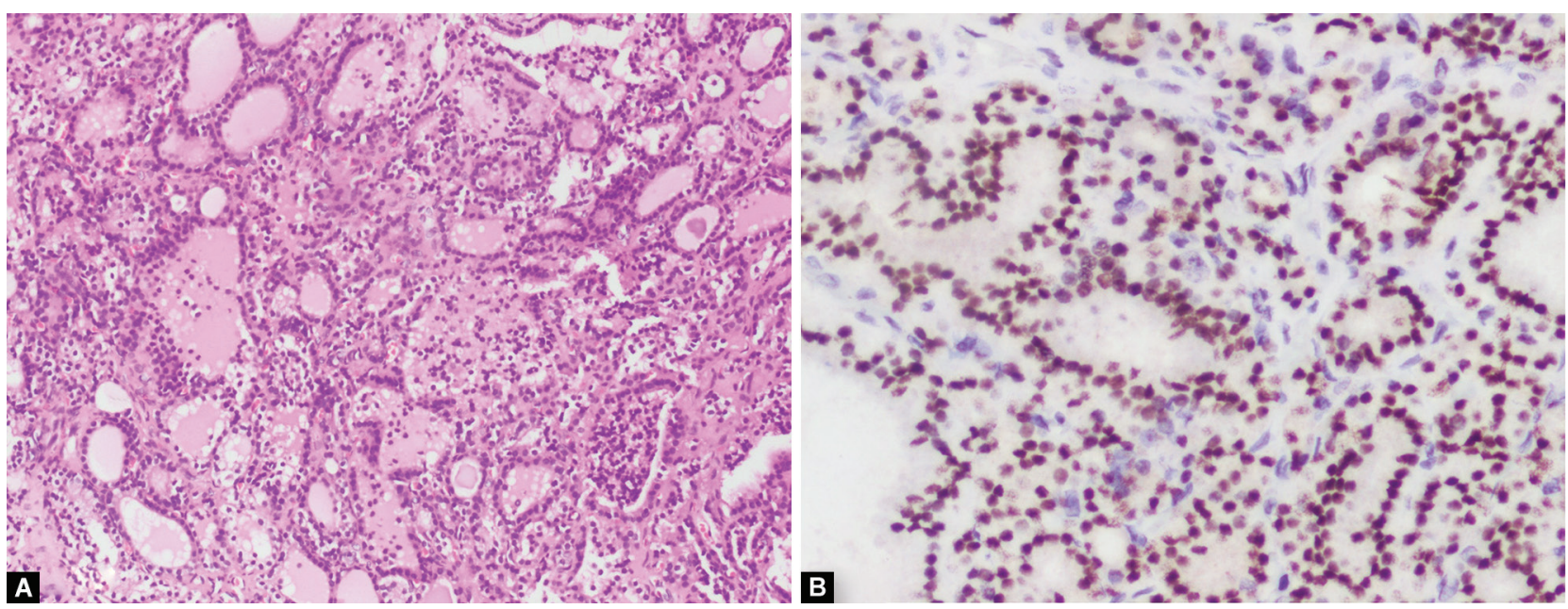

Figs 2A and B: Histopathological features depicting struma ovarii. (A) Well-circumscribed tumor composed of lobules of variably sized thyroid follicles (H\&E, 200X); (B) Immunohistochemistry study with TTF-1 showing nuclear positivity in thyroid follicles

Postoperatively, the patient recovered uneventfully but later developed subclinical hypothyroidism.

\section{Discussion}

The most common ovarian germ cell neoplasms are benign mature cystic teratomas, more commonly called dermoid cysts. On histological examination, finding thyroid tissue within them is rare, but if it composes more than $50 \%$ of the mass, then the term struma ovarii is applied. It can be asymptomatic or present as a pelvic mass in the reproductive age-group with otherwise normal parameters. The proportion of patients who present with symptoms of hyperthyroidism and can clinically be diagnosed as a case of struma ovarii is very low. A combination of struma ovarii with elevated CA-125 and gross ascites has rarely been reported and thus, in our case, gave rise to clinical suspicion of malignancy. The patient was carefully evaluated keeping the possibility of a malignant tumor in mind and underwent a staging laparotomy. The histopathology, however, gave a reassuring diagnosis of struma ovarii, which was further confirmed by immunohistochemistry.

Only about $5 \%$ of struma ovarii is found to be malignant, out of which papillary carcinoma is the most frequent variant. Immunohistochemical markers such as HBME-1, CK19, and CD56 may be used to confirm malignancy. ${ }^{2}$ This tumor is extremely uncommon, and therefore, there is paucity of specific guidelines to outline the management of such cases.

The rarity of this tumor and its resemblance to malignancy poses a major diagnostic challenge. The presence of hyperthyroidism, ascites, pseudo-Meigs' syndrome, and elevated tumor markers all contribute to the already perplexing task of diagnosing a struma ovarii. Identification of "struma pearl" on ultrasound might be helpful for diagnosis. Intracystic high-attenuation lesions suggestive of thyroid colloid have been seen on CT scan in some cases. The aid of MRI may also be taken. According to recent studies, thyroid scintigraphy may also be utilized for an accurate diagnosis. ${ }^{3}$

\section{Conclusion}

Struma ovarii can imitate ovarian malignancy clinically, especially if it is complex and found in combination with ascites and an elevated CA-125 level. Rarely, it may be associated with overt hyperthyroidism in which case the management becomes even trickier. It is important to keep in mind struma ovarii as a differential diagnosis in such a woman who is planned for surgery because a hyperthyroid crisis can occur due to surgical stress. Preoperatively, diagnosed patients may even be offered a laparoscopic approach for management and avoid major surgeries and related morbidity. Furthermore, the thyroid gland can increase in size or subclinical or clinical hypothyroidism can follow oophorectomy. Although the presentation is often vague, a high degree of clinical vigilance and appropriate aid of imaging techniques can provide more accurate preoperative diagnosis and help prevent extensive laparotomies in such patients.

\section{Clinical Significance}

This case is one of the very few that provide a description of an atypical presentation of a rare tumor, struma ovarii. A high level of clinical suspicion and appropriate knowledge may help with better preparedness before surgery and prevent extensive laparotomies in such patients.

\section{ACKnowledgment}

This research did not receive any specific grant frosm funding agencies in the public, commercial, or not-for-profit sectors. Due clearance was obtained from the Institutional Ethics Committee for this case report.

\section{References}

1. Singh $P$, Lath $N$, Shekhar $S$, et al. Struma ovarii: a report of three cases and literature review. J Midlife Health 2018;9(4):225-229. DOI: 10.4103/ jmh.JMH_53_18.

2. Zhang T, Chen P, Gao Y. Struma ovarii: a mini review. Int J Clin Exp Med 2018;11(10):10364-10371. DOI: 10.33552/WJGWH.2019.02.000532.

3. Fujiwara S, Tsuyoshi H, Nishimura T, et al. Precise preoperative diagnosis of struma ovarii with pseudo-Meigs' syndrome mimicking ovarian cancer with the combination of 131 I scintigraphy and 18F-FDG PET: case report and review of the literature. J Ovarian Res 2018;11(1):1-8. DOI: 10.1186/s13048-018-0383-2. 